\title{
Tumor-Targeting Bacteria: As Vectors, Immunotherapeutic Agents And Tumor-Targeting Probes For Cancer Detection And Therapy
}

\author{
Lihini Ranesha Weerakkody and Chamindri Witharana
}

\begin{abstract}
Cancer is the world's second leading cause of death in humans. Conventional anticancer therapies are often associated with lack of tumor specificity, failure to detect small metastases, increased resistance of tumors to anticancer drugs, and unintended adverse effects. Numerous alternative and better strategies in cancer treatment have been developed to overcome the negative effects of traditional cancer therapies. More than a century ago, William Coley, the father of cancer immounotherapy, laid the groundwork for bacterial anticancer therapy. Bacterial immunotherapy has been emerging as a potential anticancer therapy. Moreover, certain obligate and facultative anaerobic bacterial species are exploited as vectors for gene delivery to treat cancer. These genes encode for anticancer agents, cytokines, cytotoxic peptides, antiangiogenic proteins, therapeutic molecules and prodrugconverting enzymes. Genetically engineered bacterial strains of Salmonella, Bifidobacterium, Clostridium and Listeria are widely used to deliver genes in anticancer therapy since they can selectively accumulate in solid tumors with a hypoxic/necrotic core in vivo, providing appealing delivery systems to target therapeutic agents and immunomodulatory molecules to the site of tumor. Certain genetically modified bacterial species such as Bifidobacterium longum and Bacillus licheniformis have been effectively used for the enzyme/prodrug therapy for cancer. Furthermore, certain anaerobic bacteria are emerging as potential tumor markers due to the increased mobility and the selectivity in germinating and multiplying in hypoxic/anoxic environments. Many of these novel developments have been studied extensively in different experimental models of cancer and certain clinical trials are ongoing for some treatment modalities. Although favourable results have shown so far, further studies and technological innovations are required to ensure the efficacy of bacterial anticancer therapy.
\end{abstract}

Index Terms - Bacteria, cancer, gene therapy, immunotherapeutic agents.

\section{INTRODUCTION}

Cancer is one of the major causes of human death worldwide and according to the World Health Organization (WHO), the burden of cancer is increasing at an alarming rate globally [1]. Cancer is a comprehensive term for a collection of more than 100 related diseases. Malignant tumors and neoplasms are synonyms for cancer. Uncontrollable division of abnormal cells is known as cancer and these cancerous cells often invade adjoining

Published on June 30, 2020.

L. R. Weerakkody, University of Colombo, Sri Lanka.

(e-mail: vetlihini@gmail.com).

C. Witharana, University of Colombo, Sri Lanka.

(corresponding e-mail: chamindri@bmb.cmb.ac.lk) tissue and can even metastasize to distant sites. Almost all the sites of the human body are susceptible for the development of cancer.

There are over 200 different types of known cancers which affect human beings. Benign tumors are less harmful and not considered as malignant tumors. They are often treatable when compared to malignant cancers, which are highly dangerous, debilitating and life-threatening. A significant proportion of cancers can be cured, by conventional therapies such as surgery, radiotherapy or chemotherapy, especially if they are detected early. However, there are numerous limitations of these traditional treatments. Therefore, a number of novel therapeutic strategies have been developed as potential anti-cancer therapies to overcome these adverse effects of conventional anti-cancer therapies. Bacterial anti-cancer therapy is an emerging novel approach in cancer treatment [2,3].

\section{BACKGROUND}

Microorganisms, especially live bacteria have been recognized as a very potent antitumor agent that has led to the remission of certain tumors over many generations. In their experimental studies, W. Busch and F. Fehleisen observed that accidental erysipelas (Streptococcus pyogenes) infections had reduced the size of tumors in hospitalized patients [4]. In 1893, Dr. William B. Coley, a bone surgeon, started to investigate the relationship between erysipelas infection and the spontaneous regression of tumors. While reviewing the hospital records, Coley came across an interesting case, involving a patient with a sarcoma on his left cheek, who had eventually recovered completely due to the accidental exposure to streptococcal infection [5]. Dr. Coley discovered that tumor remission of cancer patients infected with erysipelas, was due to an immune response triggered by the immune system. Afterwards, he started to treat his cancer patients with live streptococcus bacteria. Dr. Coley introduced a safer vaccine in the late 1800's composed of two killed bacterial species, Streptococcus pyogenes and Serratia marcescens. This combination effectively simulated an infection with inflammation, fever, and chills without causing the real infection and it had significantly decreased the mortality rate in patients [6], [7]. This vaccine was called 'Coley's toxins' [8] and was extensively utilized around the world to treat lymphomas, bone and soft-tissue sarcomas, carcinomas, and myelomas [4], [9], [10]. Later, Dr. Coley was recognized as the 'father of cancer immunotherapy' for his significant 
contributions to the field of oncology. Coley's toxins laid the groundwork towards bacterial anticancer therapy.

\section{OVERVIEW OF BACTERIAL ANTICANCER THERAPY}

Scientists revealed that certain obligate and facultative anaerobic bacterial species such as Clostridium thrive only in hypoxic/anoxic neoplastic tissues (specifically within the core of a solid tumor), whereas they lose their viability in the well-oxygenated areas, suggesting that they are innocuous to the remaining healthy tissues of the body [11]. These findings provided the rationale to use anaerobic bacteria as effective oncolytic agents. Anaerobic bacteria and their cytotoxic effects are selectively targeted and restricted only to the cancerous tissues which are devoid of oxygen, without causing any collateral damage to the surrounding healthy tissues. Since, tumor-targeting bacteria do not destroy the entire cancerous tissue, other conventional approaches (ex: chemotherapy and radiotherapy) should be combined with the bacterial therapy. Bacterial toxins and spores have been widely used in numerous studies as anti-cancer agents [3]. Cancer vaccines play a crucial role in oncotherapy and it is based on bacterial immunotoxins [12]. Live, attenuated or genetically engineered avirulent bacteria can be used as carriers of oncolytic agents and as immunotherapeutic agents, and to provide direct oncolytic effects. Genetically manipulated bacteria for selective oncolysis, and bacterial gene-directed enzyme prodrug therapy (GDEPT) have also shown promising results in cancer therapy. This review focuses on the use of bacteria as carriers of anticancer agents and certain enzymes and as immunotherapeutic agents in cancer therapy. Furthermore, the use of anaerobic bacteria as a probe for cancer detection is also described in this review.

\section{BACTERIA AS VeCtORS For ANTI-CANCER GENE THERAPY}

Conventional anti-cancer therapies such as chemotherapy and radiotherapy have numerous drawbacks. Thus, a number of new methods have been developed as potential anticancer therapies to overcome these issues. One such method is the delivery of anti-cancer genes to the tumor site (gene therapy) [13].

In a global context, gene therapy requires the reversal of a genetic defect by adding a normal version of a faulty or absent gene to cure an underlying condition [14]. Thus, anticancer gene therapy has been mainly involved in replacing the mutated versions of proto-oncogenes and tumor suppressor genes. In addition to that, some of the genetic methods utilized are delivery of genes encoding prodrug activating enzymes, cytotoxic agents, apoptosisinducers, anti-angiogenic proteins or cell-targeted toxins to the tumor. Several prospective strategies of anticancer gene therapy include the expressing of a gene to induce apoptosis or increase tumor sensitivity to conventional chemotherapy/radiation therapy.

Even though these methods have been used, there are certain detrimental effects in these methods including the strict requirement of local administration of vectors, thus limiting their efficacy [15]. Another major problem in cancer gene therapy is the difficulty to deliver the respective gene in a target specific manner to the solid tumor. One way of overcoming these restrictions is the use of genetically modified obligate or facultative anaerobic bacteria, expressing a specific gene which has the therapeutic efficacy, only within the core of the solid tumor (Fig. 1). In this manner, the specific protein/product is only produced inside the tumor micro-environment, because the bacteria are specifically targeted to the tumor. Thus, the bacterial gene therapy plays an important role as an adjuvant therapy in oncolysis. Thus, certain bacteria serve as vectors for preferentially delivering anticancer agents, cytotoxic peptides, therapeutic proteins, apoptosis-inducers, apoptosisexecutioners or prodrug converting enzymes to tumor masses [2]. Even though bacteria are effective modes for anti-cancer therapy, there are two major drawbacks in using them. When bacteria is used at the appropriate dose to obtain the therapeutic efficacy, it causes bacteria induced toxicity and on the other hand, if the dose is reduced to prevent toxicity, it results in reduced efficacy.

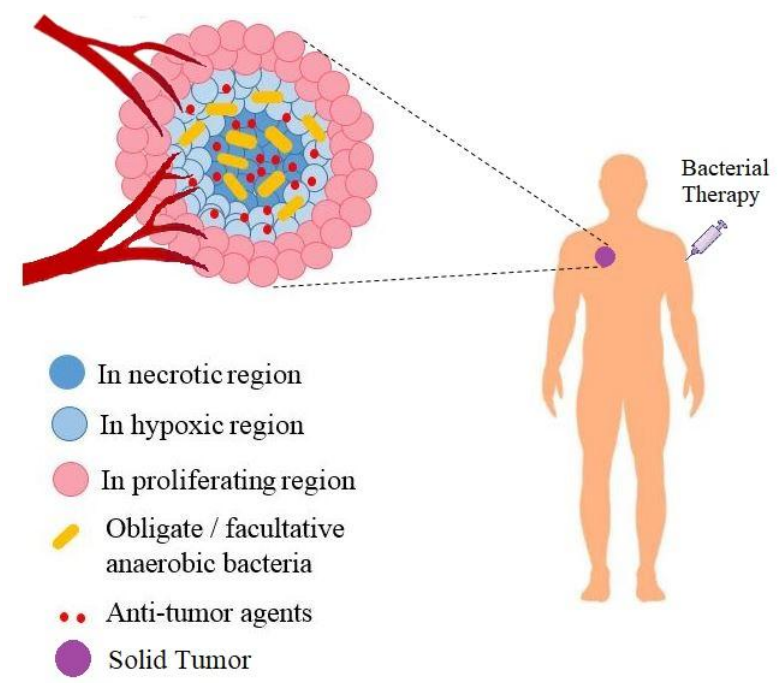

Fig. 1. Delivery of anticancer agents inside the core of a solid tumor by tumor targeting bacteria. When genetically engineered obligate or facultative anaerobic bacteria are systemically administered, they migrate far from vasculature and selectively colonize in the hypoxic/necrotic regions of the solid tumor (core of the solid tumor). Within the core of the tumor, these bacteria produce various antitumor agents (apoptotic inducers, cytotoxic agents, cytokines, tumor specific antigens, antiangiogenic agents and enzymes that convert prodrugs to active anticancer drugs) which destroy cancer cells.

\section{A. The ideal bacterial vector for anticancer gene therapy}

So far, the ideal vector for tumor-targeted gene therapy has not yet developed, however, various recombinant bacterial species have been developed and used as vectors, including Bifidobacteria [16, 17], Clostridia [18, 19] and Salmonella enterica serovar Typhimurium [20, 21] even though there are few drawbacks associated with them. The ideal vector should possess certain characteristics such as, it should be genetically traceable, non-virulent, should only replicate within the tumor mass, motility, nonimmunogenicity, and being harmless to normal tissues [15]. 


\section{B. Bacteria engineered to express tumoricidal agents}

Bacteria possess the capability to produce and deliver specific compounds; these can be artificially coupled to certain anticancer agents. Bacteria play a major role in the drug carrier field. There are various strategies employed and two predominant mechanisms have been extensively studied: the direct expression of anti-tumor proteins and transfer of eukaryotic expression vectors into infected cancer cells. Some of the anti-cancer agents expressed by genetically modified, facultative or obligate anaerobic bacteria are shown in Table I [22].

Several examples are mentioned in this review in support of bacteria used as vectors to express certain anticancer agents.

An attenuated, motile strain of Salmonella typhimurium, VNP 20009 has been genetically modified to express two apoptotic proteins, p53 and azurin in order to treat glioblastomas in mice. Xenografts of human glioblastoma brain tumors in mice were successfully treated with this recombinant strain by injecting intra-cranially at the tumor implant site. The genes encoding for these two apoptotic proteins were placed under a hypoxic promoter, pflE, which expressed these two genes only in hypoxic environments. Thus, the expression of apoptotic proteins only occurred within the tumor and induced apoptosis in cancerous cells. As Salmonella is an intracellular pathogen, it thrives inside the cancer cell, thus releases azurin into the cancer cell's cytosol, leading to apoptosis. According to the results of this experiment, survival rate has been significantly increased, no systemic toxicity, significant tumor suppression and the responders have restored the neural environment [23].

Another example for using bacteria as an effective vector for anticancer therapy is the use of a genetically modified Salmonella strain, YB1 to treat neuroblastoma. Salmonella is a facultative anaerobic bacteria, however this engineered strain is an "obligate" anaerobe, which thrive only in anaerobic/hypoxic environments. In this strain, one of the essential genes, asd, has been genetically modified to be controlled by a hypoxic conditioned promoter. The asd gene is responsible for synthesizing DAP (diaminopimelic acid), which is an essential compound in the Gram negative bacterial cell wall. Thus, in aerobic environment, such as in normal healthy tissues, there is no asd expression as the promoter is inactive, thus, leading to loss of DAP in the cell wall, which ultimately results in the lysis of the bacterium. Conversely, in the hypoxic/necrotic condition, such as a core of a solid tumor, the asd gene is expressed and DAP is synthesized, and the bacteria thrive well. Thus, this engineered strain, YB1 only multiply under hypoxic conditions (less than $0.5 \%$ oxygen) without additional DAP. This selective replication of this mutant is used to treat neuroblastoma (NB), which is a type of solid tumor in mice.

Intra-cranial fluorescent orthotopic xenograft of neuroblastoma mouse model was used in the experiment. In the YB1-treated mice with neuroblastoma xenograft, an extensive tumor regression was reported, with no side effects. The YB1 strain selectively accumulated only in the core of the tumor, whereas, the normal tissues were unaffected. Significant cell apoptosis of NB cancerous cells was observed. Thus, the mutant strain YB1, can be successfully utilized as a tool in delivering anticancer agents to treat a variety of solid tumors [24].

TABLE I: ANTI-CANCER AGENTS EXPRESSED By GENETICALLY ENGINEERED BACTERIA

\begin{tabular}{|c|c|}
\hline $\begin{array}{c}\text { Anti-cancer } \\
\text { agent }\end{array}$ & Examples \\
\hline $\begin{array}{l}\text { Apoptotic } \\
\text { Proteins }\end{array}$ & $\begin{array}{ll}\text { - } & \text { p53 } \\
\text { - } & \text { Azurin }\end{array}$ \\
\hline $\begin{array}{l}\text { Cytotoxic } \\
\text { agents }\end{array}$ & 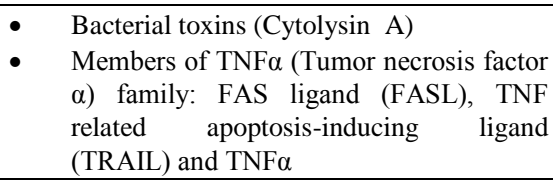 \\
\hline $\begin{array}{l}\text { Anti- } \\
\text { angiogenic } \\
\text { agents }\end{array}$ & $\begin{array}{ll}- & \text { Endostatin } \\
\text { - } & \text { Tumstatin } \\
\text { - } & \text { Thrombospondin-1 (TSP-1) } \\
\end{array}$ \\
\hline Cytokines & $\begin{array}{ll}\text { - } & \text { Interleukin (IL-2) } \\
\text { - } & \text { LIGHT } \\
\text { - } & \text { Interleukin 18 (IL-18) } \\
\text { Chemokine (C-C motif) ligand } 21 \text { (CCL } \\
\text { 21) }\end{array}$ \\
\hline Tumor antigens & 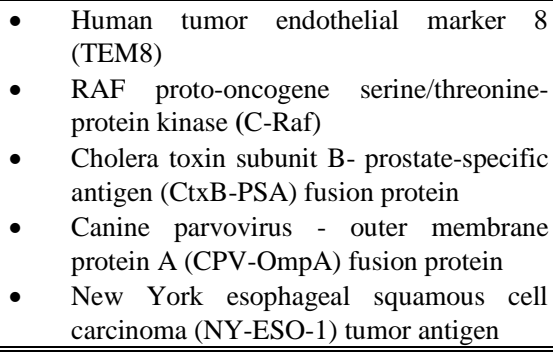 \\
\hline
\end{tabular}

Bifidobacterium longum $(B L)$ has recently been used as a delivery system for the antiangiogenic protein, tumstatin. (Tum). Tum is an endogenous angiostatin, which inhibits endothelial cell proliferation and migration. It also induces apoptosis and inhibits angiogenesis, thus preventing metastasis. Overall protein synthesis post-transcriptionally is also inhibited. $B L$ is a non-pathogenic, anaerobic bacteria which has a hypoxic metabolic characteristic, thus used as an effective gene delivering tool to solid tumors with a hypoxic core. Tum gene is expressed under pBBADs promoter of $B L$. Colon carcinoma murine model was used in the experiment and the results have shown significant antitumor effects with the Tum-expressing $B L$ treatment. Administration of $B L$ to tumor-bearing mice resulted in a strong inhibition of angiogenesis and reduced tumor growth, with a low microvessel density (MVD) than the negative control [25].

Clostridia has been used to deliver genes in anticancer therapy. Mouse tumor necrosis factor $\alpha$ (mTNF $\alpha$ is toxic to cancerous cells and it induces $\mathrm{T}$ cell mediated immunity. The gene encoding for mTNF $\alpha$ is placed under the control of RecA promoter, which gets activated by single strand breaks in DNA. Ionizing radiation causes single strand breaks in deoxyribonucleic acid (DNA) [26]. Thus, combination therapy of radiation and Clostridium-based gene therapy showed significant increase in mTNF $\alpha$ production, which led to tumor lysis [27].

\section{Carriers of bacterial enzymes (bacterially directed enzyme prodrug therapy)}

Several bacteria have been used as carriers of enzymes which can convert a non-toxic, non-functional prodrug to a toxic, active drug. Clostridial-directed enzyme-prodrug therapy (CDEPT) is one of the common strategies used so 
far in treating certain cancer types. Clostridium is an anaerobic bacteria which multiply solely in hypoxic/necrotic tissues. Thus, the gene encoding for the specific enzyme is also only expressed in hypoxic/necrotic tissues, such as the center of a solid tumor. Therefore, the prodrug is only activated within a tumor mass, and not in other healthy tissues of the body.

Several enzyme/prodrug systems are available including cytosine deaminase (CD) and nitroreductase (NR) [28] [33]. CD converts 5-fluorocytosine (5-FC) to 5-fluorouracil (5-FU), and nitroreductase (NR) converts the pro-drug, 5Aziridinyl-2,4-dinitrobenzamide to a DNA cross-linking agent. Both enzymes essentially do not have a human equivalent. CD is better than NTR, as both 5-FU and 5-FC have been approved for clinical applications. The differential toxicity between 5 -FC and 5-FU is significantly large because 5-FU is further metabolized into two inhibitors of DNA and ribonucleic acid (RNA) synthesis [34]. Certain bacterial species have been used in the enzyme/prodrug systems with significant efficacy and efficiency.

Genetically engineered Bifidobacterium longum $(B L)$ has been used in the enzyme/prodrug therapy to treat autochthomous mammary tumors in rats. A plasmid, pBLES 100-S-eCD was constructed including the Escherichia coli cytosine deaminase (eCD) gene, and the plasmid was transferred into $B L$. As BL is an anaerobic bacterium, it only proliferated within the hypoxic/necrotic zone of the tumor in rats, whereas the normal tissues and organs were unaffected. Thus, eCD was also produced only within the tumor mass, and 5-FC was converted to 5-FU within the tumor, providing a high concentration of 5-FU inside the tumor. The experimental results showed significant antitumor efficacy in rats bearing autochthomous mammary tumors, when administered with genetically modified $B L$ strain directly to the tumor or intravenously [35].

Thermophilic bacterium, Bacillus licheniformis has been recently exploited to synthesize NTR enzyme. Previously E.coli NTR gene was used in enzyme/prodrug therapies, however it was reported that there were many drawbacks of E.coli NTR enzyme. Unlike E.coli NTR enzyme, NTR enzyme derived from Bacillus licheniformis is more thermostable with high activity, which only produced the desired form, 4- hydroxylamine derivative of CB1954. Thus, Bacillus licheniformis is a suitable candidate for enzyme/prodrug therapy [36].

King and his coworkers [37] demonstrated that when a genetically engineered, attenuated strain of Salmonella typhimurium expressing an eCD (eCD), was administrated intravenously (single bolus) to tumor-bearing mice (subcutaneously implanted colon cancer), followed by intraperitoneal 5-FC injection, the tumor inhibition occurred. Mutant $S$. typhimurium had accumulated selectively inside the tumor, expressing the eCD gene and the 5-FC was effectively converted to toxic, anti-tumor 5FU. This combined therapy is known as TAPET-CD (Tumor Amplified Protein Expression Therapy- Cytosine Deaminase) and S. typhimurium was shown to be an efficient tumor-targeting bacterial species which could be used as a vehicle for gene delivering.

A pilot study was conducted by Nemunaitis and coworkers [29] in refractory cancer patients in order to evaluate the feasibility of intra-tumoral administration of TAPET-CD, followed by 5-FC injection. Salmonella typhimurium expressing an E.coli $\mathrm{CD}$ was observed in high levels in the tumor and significant levels of therpaeutic 5-FU was also observed within the tumor mass. The intra-tumoral colonization of Salmonella typhimurium makes itself a suitable gene delivery vehicle in cancer therapy without any adverse effects.

Another study was performed to demonstrate the efficacy of TAPET-CD plus 5-FC therapy in tumor regression in C38-tumor transplanted mice. Neither 5-FC treatment alone nor 5-FU treatment alone could reduce the size of the tumor mass. However, the combination of TAPET-CD and 5-FC therapy showed significant tumor regression [38].

Fox and co-workers [39] cloned the CD gene of E. coli into a clostridial expression vector and transformed this into $C$. beijerincki. High amounts of the $\mathrm{CD}$ enzyme were detected in the bacterial culture medium and the sensitivity of murine EMT6 carcinoma cells to 5-FU showed a significant increase in 500-fold. Quantitative in vitro analyses of $C$. acetobutylicum, which was engineered to express $\mathrm{CD}$, showed biologically active protein in lysates and supernatants of the transgenic bacteria [40]. Furthermore, CD activity was detected in the tumour mass after intratumoural administration of recombinant $C$. acetobutylicum spores to Wistar Albino Glaxo from Rijswijk (WAG/Rij) rats bearing rhabdomyosarcomas [40, 41].

\section{As IMMUNOTHERAPEUTIC AGENTS}

Immunotherapy is a novel treatment strategy used in cancer therapy. It is proven to be effective than conventional chemotherapy and radiotherapy with less adverse effects. Since tumors are immunogenic by nature, the presence of a tumor stimulates the immune system to eliminate it. Thus, immunotherapy facilitates the stimulation of the immune system to destroy malignant cells. However, tumors possess certain mechanisms to evade the immune responses by developing tolerance as they are weakly immunogenic and in certain instances, body itself recognizes them as selfantigens. The inherent antigenicity of the tumor cells is enhanced by the use of non-pathogenic, anaerobic bacteria, thus making bacteria as a promising agent to be used as immunotherapeutic agents [42].

Avogadri and coworkers [43], in their experiment, demonstrated that attenuated, but invasive $S$. typhimurium could eliminate tumor cells from the host, along with the anti-Salmonella vaccination. $S$. typhimurium was successfully used in infecting the melanoma tumor cells both in vivo and in vitro, and the subsequent immune responses were observed. Upon intra-tumoral $S$. typhimurium administration, melanoma cells presented antigenic determinants of bacterial origin which were targets for the anti-Salmonella specific T cells. Prior to Salmonella infection, anti-Salmonella vaccination was also given to the tumor-bearing mice. Tumor-bearing mice which had received the vaccine and the intra-tumoral injection of $S$. typhimurium were free of tumor cells after a certain period and induced tumor specific immune response. Furthermore, S. typhimurium showed indirect toxicity towards tumor 
cells.

The property of selective colonization of attenuated $S$. typhimurium, encoding the murine cytokine IL-2 was utilized in immunotherapy in cancer treatment. Mice with the implants of melanoma cells were treated with IL-2 encoding $S$. typhimurium and subsequent tumor regression was observed. It was reported that cytokines have the potential to modulate immunity to infection. Cytokine expression in the bacterial strains is regulated by the nirB promoter, whose function is significantly enhanced under hypoxic conditions, such as a core of a solid tumor. Thus, the specific cytokine is produced at high concentrations only within the tumor mass [44].

Angiogenesis is one of the prominent features in tumor growth. In tumor endothelial cells, TEM8 (Tumor Endothelial Marker 8) is dominantly expressed. In a study conducted by Ruan and coworkers [45], a xenogenic DNA vaccine encoding human TEM8 carried by attenuated $S$. typhimurium was constructed. This vaccine was give orally to tumor bearing mice. Following vaccination, marked CD8+ cytotoxic $\mathrm{T}$ cell response was observed and according to the results of the study, life span of mice was increased. Furthermore, angiogenesis was suppressed and no adverse effects were reported. Thus, TEM8 encoding Salmonella vaccine demonstrated the potential in antiangiogenesis immunotherapy strategy.

Listeria monocytogenes has been utilized as an effective carrier for Her-2/neu, a member of the epidermal growth factor receptor family which is present in an active form in human breast tumors. Listeria monocytogenes, being an intra-cellular bacterium, can stimulate both innate and cellmediated immunity in hosts. Live, recombinant Listeria monocytogenes, expressing a single fragment of the Her$2 /$ neu was used as a vaccine for tumor bearing mice. According to the results of the experiment, regression of the tumors, slowing and inhibition of tumor growth and complete eradication of the established tumors in preclinical mouse tumor models were observed [46].

Another study demonstrated the efficacy of using a Listeria monocytogenes (LM) based vaccine in treating metastatic breast cancer. An attenuated strain of Listeria monocytogenes was utilized in a vaccine to eradicate all metastases and almost the entire primary tumor in an aggressive mouse tumor model 4T1. This remarkable eradication was due to direct killing by Listeria monocytogenes and by cytotoxic $\mathrm{T}$ lymphocytes (CTL) responses against Listeria monocytogenes antigens, including the truncated listeriolysin O (LLO).This LMbased vaccine has been successfully used in eliminating metastatic breast cancers [47].

Loeffler and coworkers [48] have successfully demonstrated the capability of a recombinant strain of attenuated $S$. typhimurium in inhibiting the growth of primary tumors and halting the dissemination of pulmonary metastases in a vast variety of murine models. Attenuated $S$. typhimurium has been genetically modified to express a specific cytokine, known as LIGHT, which promotes tumor rejection. When the mutant bacterial species was administered to murine tumor models with mouse carcinoma cell lines in immunocompetent mice, antitumor activity was observed and there was no evidence of toxicity. All these experimental findings indicate the potential use of avirulent bacteria as cancer immunotherapeutic agents.

\section{BACTERIA AS A TOOl For CANCER Diagnosis}

\section{A. Anaerobic bacteria as tumor markers}

Various methods have been used to detect the presence of a tumor and to locate the tumor exactly. Basically two types of non-bacterial material are used as tumor markers to locate the tumor; viral vectors and non-viral vectors [49] - [52] (Table II).

All these above mentioned tumor markers have drawbacks to a certain extent such as certain methods are incapable of detecting small metastases. Thus, anaerobic bacteria is emerging as a promising tool as a tumor marker due to the increased mobility and the selectivity in germinating and multiplying in hypoxic/anoxic environments. This strategy is relatively effective, simple, and direct and very useful in detecting both large and small metastases too, in addition to the primary tumor, which is essential for a positive prognosis. Following the administration of the marker, the location of the tumor is detected via bioluminescence, fluorescence, magnetic resonance imaging (MRI) and positron emission tomography (PET) [53]. To detect the location of the tumor, following the bacterial marker administration, light, MRI or positron emission tomography have been currently used [22], [54].

TABLE II: EXAMPLES FOR NON-BACTERIAL TUMOR MARKERS

\begin{tabular}{|c|c|}
\hline Viral vectors & Non-viral vectors \\
\hline Adeno-associated virus & MicroRNA \\
\hline $\begin{array}{c}\text { Herpes simplex virus } \\
\text { (HSV)-1 }\end{array}$ & Short hairpin (sh)RNA \\
\hline HSV amplicon & Small interfering (si)RNA \\
\hline Sindbis & $\begin{array}{c}\text { Oligodeoxynucleotides } \\
\text { (ODNs) }\end{array}$ \\
\hline Poliovirus replicon & Therapeutic DNA \\
\hline \multicolumn{2}{|l|}{$\begin{array}{c}\text { Lentivirus/Moloney murine } \\
\text { leukemia virus } \\
\end{array}$} \\
\hline Adenovirus & \\
\hline
\end{tabular}

VNP20009 expressing HSV-tk (Herpes Simplex Virus type 1 - thymidine kinase) reporter gene is currently used as a tumor marker to detect the location of tumors successfully. This is a non-invasive method to detect primary tumors and small metastases. This specific strain selectively colonize within the murine tumor models and showed a significant accumulation of a radio-labeled nucleoside analogue, FIAU (2'-fluoro-2'-deoxy-5-iodouracil-B-D-arabinofuranoside) inside the tumor tissue. This finding was observed by PET imaging system. According to the PET images, even the small metastases could be identified [55]. Tjuvajev and coworkers [21] have performed a preliminary study to demonstrate localization of radiolabeled ${ }^{14} \mathrm{C}$-FIAU in tumorbearing mice pretreated with mutant Salmonella strain, VNP20009 expressing the reporter gene, HSVI-tk. Currently HSV1-tk enzyme is considered as the most exclusively used self-destructive agent for gene therapy of cancer. ${ }^{14} \mathrm{C}$-FIAU is one of the substrates of HSV1-tk enzyme and it is phosphorylated by HSV1-tk enzyme. When the mutant 
Salmonella strain, VNP20009 expressing the HSV1-tk gene was administered to tumor-bearing mice, the bacterial organisms selectively localized and replicated inside the hypoxic/necrotic core of the tumor. The radiolabeled ${ }^{14} \mathrm{C}$ FIAU is thus selectively phosphorylated by HSV1-tk enzyme and were trapped in the mutant Salmonella strain, VNP20009 expressing the HSVI-tk gene inside the tumor tissue. The ${ }^{14} \mathrm{C}$-FIAU radioactivity and bacterial count data strongly indicate a Salmonella (TK)-dependent ${ }^{14} \mathrm{C}$-FIAU accumulation in the tumor tissue than the normal healthy tissue.

Certain bacterial species have been used to detect both primary tumors and previously unknown metastases effectively which is crucial for prognosis. Thus, these bacterial species could be developed further to detect tumors and can be used as tumor markers as shown in Table III [56] $-[59]$.

TABLE III: EXAMPLES FOR BACTERIAL TUMOR MARKERS

\begin{tabular}{ll}
\hline \hline Bacterial species & Genetic modification which aids detection \\
\hline $\begin{array}{l}\text { Salmonella } \\
\text { typhimurium }\end{array}$ & $\begin{array}{l}\text { Transformed with plasmid pLITE201 (a } \\
\text { plasmid which has the lac promoter fused to the } \\
\text { genes of } \text { Photorhabdus luminescens) containing } \\
\text { luxCDABE }\end{array}$ \\
\hline $\begin{array}{l}\text { Listeria } \\
\text { monocytogenes }\end{array}$ & $\begin{array}{l}\text { Transformed with plasmid pSOD-GFP,carrying } \\
\text { the GFP (Green Fluorescent Protein) gene } \\
\text { construct under the control of the SOD } \\
\text { promoter }\end{array}$ \\
\hline Vibrio cholerae & $\begin{array}{l}\text { Transformed with plasmid pLITE201 (a } \\
\text { plasmid which has the lac promoter fused to the } \\
\text { genes of Photorhabdus luminescens) containing } \\
\text { luxCDABE }\end{array}$ \\
\hline Vibrio cholerae & $\begin{array}{l}\text { Transformed with a luxCDABE bacterial } \\
\text { luciferase expression cassette }\end{array}$ \\
\hline $\begin{array}{l}\text { Salmonella } \\
\text { typhimurium }\end{array}$ & $\begin{array}{l}\text { Transformed with a luxCDABE bacterial } \\
\text { luciferase expression cassette }\end{array}$ \\
\hline Escherichia coli & Transformed with a luxCDABE bacterial \\
& luciferase expression cassette \\
\hline \hline
\end{tabular}

\section{CONCLUSION}

Currently, cancer is the second cause of death of humans globally despite the various cancer treatments available. Novel therapies have arisen in the field of oncology; immunotherapy has emerged as a potential option in the battle against cancer, stimulating the immune system by inducing adaptive and innate immune responses. Certain genetically modified anaerobic, non-pathogenic bacterial species have been used for cancer immunotherapy as these bacterial therapies inherit many unique mechanisms for treating cancer which are impossible to achieve with conventional therapies. Numerous studies such as in vitro and in vivo studies of tumor-bearing laboratory animals, phase 1 clinical trials, have been performed to investigate the potential of genetically engineered bacterial species for cancer immunotherapy. Furthermore, certain bacteria have been used as carriers of anticancer agents including cytotoxic agents and anti-angiogenic agents. However, certain limitations associated with bacterial therapy have been identified and further investigations have been carried out to overcome these limitations of bacterial usage and to further improve the therapeutic potential of bacterial strains.
Since, cancer is a complex, chronic multifactorial disease, a single treatment modality is ineffective, thus, a combination of different therapies is essential. Bacterial therapy is combined with conventional therapies such as chemotherapy and radiotherapy, in order to treat cancer patients successfully with positive outcomes and to improve their quality of life. Usage of attenuated bacterial strains as cancer immunotherapeutic tools is a promising way to save many lives of cancer patients. Furthermore, bacterial species are engineered to produce numerous anticancer agents, specifically in the hypoxic tumor micro-environment. However, further investigations are required to enhance the efficacy and safety of these novel treatment strategies as there are still questions about the safety of these approaches.

\section{REFERENCES}

[1] Cancer, World Health Organization. https://www.who.int/healthtopics/cancer\#tab=tab_1. (Accessed June 5, 2020).

[2] S. Patyar, R. Joshi, B. D. S. Prasad, A. Prakash, B. Medhi and B. K. Das. (March 2010). Bacteria in cancer therapy: a novel experimental strategy. $J$ Biomed Sci. 17(1). pp. 21. Available: https://doi.org/10.1186/1423-0127-17-21.

[3] L. R. Weerakkody and C. Witharana. (October 2019). The role of bacterial toxins and spores in cancer therapy. Life Sci. 235:116839. Available: https://doi.org/10.1016/j.lfs.2019.116839.

[4] H. C. Nauts, "The beneficial effects of bacterial infections on host resistance to cancer. End results in 449 cases. A study and abstracts in the world medical literature (1775 - 1980) and personal communications", in Monograph no.8, 22 ${ }^{\text {nd }}$ ed. Cancer Research Institute, 1980.

[5] W. B. Coley. (May 1893). The treatment of malignant tumors by repeated inoculations of erysipelas. With a report of ten original cases. Am J Med Sci. 10. pp. 487-511. Available: https://doi:10.1001/jama.1893.02420490019007.

[6] M. A. Richardson, T. Ramirez, N. C. Russell and L. A. Moye. (May 1999). Coley toxins immunotherapy: A retrospective review. Altern Ther Health Med. 5(3). pp. 42-47. Available: PMID: 10234867.

[7] L. R. Zacharski and V. P. Sukhatme. (March 2005). Coley's toxin revisited: Immunotherapy or plasminogen activator therapy of cancer? J Thromb Haemost. 3(3). pp. 424-427. Available: https://doi:10.1111/j.1538-7836.2005.01110.x.

[8] W. B. Coley. (August 1898). The treatment of inoperable sarcoma with the mixed toxins of erysipelas and bacillus prodigiosus: Immediate and final results in one hundred and forty cases. J Am Med Assoc. XXXI(9). pp. 456-465. Available: http:// doi:10.1001/jama.1898.92450090022001g.

[9] W. B. Coley. (May 1906). The treatment of inoperable sarcoma with the mixed toxins of Erysipelas and the Bacillus Prodigiosus. Lancet. 167(4316). pp. 1407-1408. Available: https://doi.org/10.1016/S01406736(00)68280-6

[10] H.C. Nauts, W. E. Swift and B. L. Coley. (April 1946). The treatment of malignant tumors by bacterial toxins as developed by the late William B. Coley, M.D., Reviewed in the light of modern research. Cancer Res. 6(4). pp. 205-216. Available: PMID: 21018724.

[11] R. A. Malmgren and C. C. Flanigan. (August 1955). Localization of the vegetative form of Clostridium tetani in mouse tumors following intravenous spore administration. Cancer Res. 15(7). pp. 473-478. Available: PMID: 13240693

[12] E. A. Carswell, L. J. Old, R. L. Kassel, S. Green, N. Fiore and B. Williamson. (September 1975). An endotoxin-induced serum factor that causes necrosis of tumors. Proc Natl Acad Sci USA. 72(9). pp. 3666-3670. Available: https://doi.org/10.1073/pnas.72.9.3666.

[13] J. A. Roth and R. J. Cristiano. (January 1997). Gene therapy for cancer: what have we done and where are we going? J Natl Cancer Inst. 89(1). pp. 21-39. Available: https://doi.org/10.1093/jnci/89.1.21.

[14] T. Friedmann. (October 1992). A brief history of gene therapy. Nat Genet. 2(2). pp. 93-98. Available: https://doi.org/10.1038/ng1092-93.

[15] R. M. Ryan, J. Green and C. E. Lewis. (January 2006). Use of bacteria in anti-cancer therapies. Bioessays. 28(1). pp.84-94. Available: https://doi.org/10.1002/bies.20336.

[16] K. Yazawa, M. Fujimori, J. Amano, Y. Kano and S. Taniguchi. (February 2000). Bifidobacterium longum as a delivery system for cancer gene therapy: selective localization and growth in hypoxic 
tumors. Cancer Gene Ther.7(2). pp. 269-274. Available: https://doi.org/10.1038/sj.cgt.7700122.

[17] X. Li, G. F. Fu, Y. R. Fan, W. H. Liu, X. J. Liu, J. J. Wang and G. X. $\mathrm{Xu}$. (February 2003). Bifidobacterium adolescentis as a delivery system of endostatin for cancer gene therapy: Selective inhibitor of angiogenesis and hypoxic tumor growth. Cancer Gene Ther. 10(2). pp.105-111. Available: https://doi.org/10.1038/sj.cgt.7700530.

[18] J.R. Möse and G. Möse. (February 1964). Oncolysis by Clostridia. I. Activity of Clostridium butyricum (M-55) and other nonpathogenic Clostridia against the Ehrlich Carcinoma. Cancer Res. 24(2). pp. 212216. Available: PMID: 14115686.

[19] S. Nuyts, L. Van Mellaert, S. Barbe, E. Lammertyn, J. Theys, W. Landuyt, et al.(October 2001). Insertion or deletion of the cheo box modifies radiation inducibility of Clostridium promoters. Appl Environ Microbiol. 67(10). pp. 4464-4470. Available: https://doi.org/10.1128/aem.67.10.4464-4470.2001.

[20] J. M. Pawelek, K. B. Low and D. Bermudes. (October 1997). Tumortargeted Salmonella as a novel anticancer vector. Cancer Res. 57(20). pp. 4537-4544. Available: PMID: 9377566

[21] J. Tjuvajev, R. Blasberg, X. Luo, L. M. Zheng, I. King and D. Bermudes. (July 2001). Salmonella-based tumor-targeted cancer therapy: Tumor amplified protein expression therapy (TAPETTM) for diagnostic imaging. J Control Release. 74(1-3). pp. 313-315. Available: https://doi.org/10.1016/s0168-3659(01)00340-6

[22] N. S. Forbes. (November 2010). Engineering the perfect (bacterial) cancer therapy. Nat Rev Cancer. 10(11). pp. 785-794. Available: https://dx.doi.org/10.1038\%2Fnrc2934

[23] N. Mehta, J. G. Lyon, K. Patil, N. Mokarram, C. Kim and R. V. Bellamkonda. (December 2016). Bacterial carriers for glioblastoma therapy. Mol Ther Oncolytics.4. pp. 1-17. Available: https://doi.org/10.1016/j.omto.2016.12.003.

[24] B. T. Ning, B. Yu, S. Chan, J. L. Chan, J. D. Huang and G. C. Chan. (June 2017). Treatment of neuroblastoma with an engineered "Obligate" anaerobic Salmonella typhimurium strain YB1. J Cancer. 8(9). pp. 1609-1618. Available: https://doi.org/10.7150/jca.18776.

[25] C. Wei, A. Y. Xun, X. X. Wei, J. Yao, J. Y. Wang, R. Y. Shi, et al. Bifidobacteria expressing tumstatin protein for antitumor therapy in tumor-bearing mice. (June 2016). Technol Cancer Res Treat.15(3). pp. 498-508. Available: https://doi.org/10.1177/1533034615581977.

[26] S. Nuyts, L. Van Mellaert, J. Theys, W. Landuyt, E. Bosmans, J. Anné and P. Lambin. (August 2001). Radio-responsive recA promoter significantly increases $\mathrm{TNF} \alpha$ production in recombinant clostridia after 2 Gy irradiation. Gene Ther. 8(15). pp. 1197-1201. Available: https://doi.org/10.1038/sj.gt.3301499.

[27] S. Nuyts, J. Theys, W. Landuyt, L. Van Mellaert, P. Lambin and J. Anné. (March-April 2001). Increasing specificity of anti-tumor therapy: Cytotoxic protein delivery by non-pathogenic clostridia under regulation of radio-induced promoters. Anticancer Res. 21(2A) pp. 857-861. Available: PMID: 11396175.

[28] J. Theys, W. Landuyt, S. Nuyts, L. Van Mellaert, A. Van Oosterom, P. Lambin and Anné J. (April 2001). Specific targeting of cytosine deaminase to solid tumors by engineered Clostridium acetobutylicum. Cancer Gene Ther. 8(4). pp. 294-297. Available: https://doi.org/10.1038/sj.cgt.7700303.

[29] J. Nemunaitis, C. Cunningham, N. Senzer, J. Kuhn, J. Cramm, C. Litz, et al. (October 2003). Pilot trial of genetically modified, attenuated Salmonella expressing the E.coli cytosine deaminase gene in refractory cancer patients. Cancer Gene Ther. 10(10). pp. 737-744. Available: https://doi.org/10.1038/sj.cgt.7700634.

[30] S.C. Liu, G. O. Ahn, M. Kioi, M. J. Dorie, A. V. Patterson and J. M. Brown. (October 2008). Optimized clostridium-directed enzyme prodrug therapy improves the antitumor activity of the novel DNA cross-linking agent PR-104. Cancer res. 68(19). pp. 7995-8003. Available: https://doi.org/10.1158/0008-5472.can-08-1698.

[31] J. Stritzker, S. Pilgrim, A. A. Szalay and W. Goebel. (April 2008). Prodrug converting enzyme gene delivery by L. monocytogenes. BMC Cancer. 8. pp. 94. Available: https://doi.org/10.1186/14712407-8-94.

[32] L. Green, M. Storey, E. Williams, A. Patterson, J. Smaill, J. Copp and D. Ackerley. (September 2013). The flavin reductase MsuE is a novel nitroreductase that can efficiently activate two promising nextgeneration prodrugs for gene-directed enzyme prodrug therapy Cancers. 5(4). pp. 985-997. Available: https://dx.doi.org/10.3390\%2Fcancers5030985.

[33] A. M. Kubiak and N. P. Minton. (Mayy 2015). The potential of clostridial spores as therapeutic delivery vehicles in tumour therapy. Res Microbiol. 166(4). pp. 244-254. Available: https://doi.org/10.1016/j.resmic.2014.12.006.

[34] A. Polak, E. Eschenhof, M. Fernex and H. J. Scholer. (1976) Metabolic studies with 5-Fluorocytosine-6-14C in mouse, rat, rabbit, dog and man. Chemotherapy. 22(3-4). pp. 137-153. Available: https://doi.org/10.1159/000221923.

[35] T. Sasaki, M. Fujimori, Y. Hamaji, Y. Hama, K. Ito, J. Amano and S. Taniguchi. (July 2006). Genetically engineered Bifidobacterium longum for tumor-targeting enzyme-prodrug therapy of autochthonous mammary tumors in rats. Cancer Sci. 97(7). pp. 649657. Available: https://doi.org/10.1111/j.1349-7006.2006.00221.x.

[36] C. D. Emptage, R. J. Knox, M. J. Danson and D. W. Hough. (January 2009). Nitroreductase from Bacillus licheniformis: A stable enzyme for prodrug activation. Biochem Pharmacol.77(1). pp. 21-29. Available: https://doi.org/10.1016/j.bcp.2008.09.010.

[37] I. King, D. Bermudes, S. Lin, M. Belcourt, J. Pike, K. Troy, et al. (Jul 2002). Tumor-targeted Salmonella expressing cytosine deaminase as an anticancer agent. Hum gene ther.13(10). pp. 1225-1233. Available: https://doi.org/10.1089/104303402320139005.

[38] I. King, M. Itterson and D. Bermudes. (2009). Tumor-targeted Salmonella typhimurium overexpressing cytosine deaminase: A novel, tumor-selective therapy. Methods Mol Biol. 542. pp. 649-659. Available: https://doi.org/10.1007/978-1-59745-561-9_33.

[39] M. E. Fox, M.J. Lemmon, M. L. Mauchline, T. O. Davis, A. J. Giaccia, N.P. Minton and J. M. Brown. (February 1996). Anaerobic bacteria as a delivery system for cancer gene therapy: in vitro activation of 5-fluorocytosine by genetically engineered clostridia Gene ther. 3(2). pp. 173-178. Available: PMID: 8867865.

[40] J. Theys, A. W. Landuyt, S. Nuyts, L. Van Mellaert, P. Lambin and J. Anné. (2001). Clostridium as a tumor-specific delivery system of therapeutic proteins. Cancer Detect Prev. 25(6). pp. 548-557. Available: PMID: 12132875

[41] J. Theys, W. Landuyt, S. Nuyts, L. Van Mellaert, E. Bosmans, A. Rijnders, et al. (February 2001). Improvement of Clostridium tumour targeting vectors evaluated in rat rhabdomyosarcomas. FEMS Immunol Med Microbiol.30(1). pp. 37-41. Available: https://doi.org/10.1111/j.1574-695x.2001.tb01547.x.

[42] J. A. Xu, X. Liu, S. Zhou and M. Q. Wei. (April 2009). Combination of immunotherapy with anaerobic bacteria for immunogene therapy of solid tumours. Gene Ther Mol Biol. 13. pp. 36-52.

[43] F. Avogadri, C. Martinoli, L. Petrovska, C. Chiodoni, P. Transidico, V. Bronte, et al. (May 2005). Cancer immunotherapy based on killing of Salmonella-infected tumor cells. Cancer Res. 65(9). pp. 3920 3927. Available: https://doi.org/10.1158/0008-5472.can-04-3002.

[44] B.K. Al-Ramadi, M. J. Fernandez-Cabezudo, H. El-Hasasna, S. AlSalam, S. Attoub, D. Xu and S. Chouaib. (September 2008). Attenuated bacteria as effectors in cancer immunotherapy. Ann NY Acad Sci.1138. pp. 351-357. Available: https://doi.org/10.1196/annals.1414.036.

[45] Z. Ruan, Z. Yang, Y. Wang, H. Wang, Y. Chen, X. Shang, et al. (June 2009). DNA vaccine against tumor endothelial marker 8 inhibits tumor angiogenesis and growth. $J$ Immunother. 32(5). pp. 486-491. Available: https://doi.org/10.1097/cji.0b013e3181a1d134.

[46] L. M. Wood, P. D. Guirnalda, M. M. Seavey and Y. Paterson. (2008). Cancer immunotherapy using Listeria monocytogenes and listerial virulence factors. Immunol Res. 42(1-3). pp. 233-245. Available: https://doi.org/10.1007/s12026-008-8087-0.

[47] S. H. Kim, F. Castro, Y. Paterson and C. Gravekamp. (July 2009). High efficacy of a Listeria-based vaccine against metastatic breast cancer reveals a dual mode of action. Cancer res. 69(14). pp. 58605866. Available: https://doi.org/10.1158/0008-5472.can-08-4855.

[48] M. Loeffler, G. Le'Negrate, M. Krajewska and J. C. Reed. (July 2007). Attenuated Salmonella engineered to produce human cytokine LIGHT inhibit tumor growth. Proc Natl Acad Sci USA. 104(31). pp. $12879-12883$ https://dx.doi.org/10.1073\%2Fpnas.0701959104

Available:

9] C. E. Thomas, A. Ehrhardt and M. A. Kay. (May 2003). Progress and problems with the use of viral vectors for gene therapy. Nat Rev Genet. 4(5). pp. 346-358. Available: https://doi.org/10.1038/nrg1066.

[50] M. Morille, C. Passirani, A. Vonarbourg, A. Clavreul and J. P. Benoit. (August - September 2008). Progress in developing cationic vectors for non-viral systemic gene therapy against cancer Biomaterials. 29(24-25). pp. 3477-3496. Available: https://doi.org/10.1016/j.biomaterials.2008.04.036.

[51] S. R. Ji, C. Liu, B. Zhang, F. Yang, J. Xu, J. Long, et al. (August 2010). Carbon nanotubes in cancer diagnosis and therapy. Biochim Biophys Acta. 1806(1). pp. 29-35. Available: https://doi.org/10.1016/j.bbcan.2010.02.004.

[52] C. M. McCrudden and H. O. Mccarthy, "Cancer gene therapy - Key biological concepts in the design of multifunctional non-viral delivery systems", in Gene Therapy - Tools and potential applications, Francisco Martin Molina, IntechOpen, 2013. Available: http:// doi.org/10.5772/54271. 
[53] C. Ptak and A. Petronis. (2008). Epigenetics and complex disease: from etiology to new therapeutics. Annu Rev Pharmacol Toxicol. 48 pp. 257-276. Available: https://doi.org/10.1146/annurev.pharmtox.48.113006.094731.

[54] R. Van der Meel, W. M. Gallagher, S. Oliveira, A. E. O'Connor, R. M. Schiffelers and A. T. Byrne. (February 2010). Recent advances in molecular imaging biomarkers in cancer: application of bench to bedside technologies. Drug Discov Today. 15(3-4). pp. 102-114. Available: https://doi.org/10.1016/j.drudis.2009.12.003.

[55] S. A. Soghomonyan, M. Doubrovin, J. Pike, X. Luo, M. Ittensohn, J. D. Runyan, et al. (January 2005). Positron emission tomography (PET) imaging of tumor-localized Salmonella expressing HSV1-TK. Cancer Gene Ther. 12(1). pp. 101-108. Available: https://doi.org/10.1038/sj.cgt.7700779.

[56] K. Flentie, B. Kocher, S. T. Gammon, D. V. Novack, J. S. McKinney and D. Piwnica-Worms. (July 2012). A bioluminescent transposon reporter-trap identifies tumor-specific microenvironment-induced promoters in Salmonella for conditional bacterial-based tumor therapy. Cancer Discov. 2(7). pp. 624-637. Available: https://doi.org/10.1158/2159-8290.cd-11-0201.

[57] J. J. Min, H. J. Kim, J. H. Park, S. Moon, J. H. Jeong, Y. J. Hong, et al. (Januaryy - February 2008). Noninvasive real-time imaging of tumors and metastases using tumor-targeting light-emitting Escherichia coli. Mol Imaging Biol. 10(1). pp. 54-61. Available: https://doi.org/10.1007/s11307-007-0120-5.

[58] Y. A. Yu, T. Timiryasova, Q. Zhang, R. Beltz and A. A. Szalay. (November 2003). Optical imaging: Bacteria, viruses, and mammalian cells encoding light-emitting proteins reveal the locations of primary tumors and metastases in animals. Anal Bioanal Chem. 377(6). pp. 964-972. Available: https://doi.org/10.1007/s00216-003-2065-0.

[59] Y. A. Yu, S. Shabahang, T. M. Timiryasova, Q. Zhang, R. Beltz, I Gentschev, et al. (March 2004). Visualization of tumors and metastases in live animals with bacteria and vaccinia virus encoding light-emitting protein. Nat Biotechnol.22(3).pp.313-320.Available: slksdfhttps://doi.org/10.1038/nbt937.

Lihini Ranesha Weerakkody completed her MSc in Biochemistry and Molecular Biology, University of Colombo, Colombo, Sri Lanka (2019) and BVSc in Veterinary Science, University of Peradeniya, Sri Lanka (2016). Her research interests include anticancer therapies, molecular basis of cancer and veterinary medicine. She is currently working as a Lecturer and her subject area is Biochemistry. She is also working as a professional Veterinary Surgeon since 2017.

Chamindri Witharana completed her $\mathrm{PhD}$ in Micro and Molecular Biology, University of Giessen (Justus Liebig University) Giessen, Germany (2013), MPhil in Biological Sciences and Biochemistry, School of Environmental Sciences, University of Wales, Swansea, United Kingdom (2009) and BSc (Hons) in Chemistry (Sp.), University of Colombo, Colombo, Sri Lanka (2006). Her main research interest is molecular and genetic basis of cancer

She is a Senior Lecturer in Biochemistry and Molecular Biology at the Faculty of Medicine, University of Colombo, Sri Lanka. She has provided her expertise in Molecular Biology at both national and international level. 